\title{
Programas específicos de tratamiento en las prisiones españolas: control de la agresión sexual, atención integral a enfermos mentales y unidades terapéuticas y educativas. ${ }^{1}$
}

Specific treatment programs in Spanish prisons: sexual assault control, integral attention to mentally-ill persons and therapeutic and educational units.

Virginia García López

Psicóloga del Centro

Penitenciario de Mujeres de

Alcalá de Guadaíra (Sevilla)

virginia_gl@hotmail.com

\section{Resumen}

El tratamiento penitenciario es, junto a la seguridad de las prisiones, uno de los pilares necesarios para poder cumplir el mandato constitucional recogido en el art. 25.2 de la CE. En la actualidad existe una gran variedad de programas para intervenir sobre las carencias y necesidades de los internos/as, por ejemplo de drogodependencias, agresores sexuales, conductas violentas, ludopatía, entre otros. Este artículo pretende acercar un poco más el funcionamiento de las prisiones al lector. Nos centraremos en explicar brevemente tres programas de tratamiento, el Programa de Control de la Agresión Sexual (PCAS), el Programa de Atención Integral de Enfermos Mentales (PAIEM) y las Unidades Terapéuticas y Educativas (UTEs). Por último, plantearemos algunas conclusiones respecto al tema que nos ocupa y también algunas de las limitaciones, así como posibles soluciones.

Palabras clave: tratamiento, prisión, programas, agresores sexuales, trastorno mental, drogas.

${ }^{1}$ Recibido: 9/06/2018 Evaluado: 02/07/2018 Aceptado: 05/05/2019 


\begin{abstract}
The penitentiary treatment, is, together with security in prisons, one of the necessary pillars to be able to fulfill the constitutional mandate included in the 25.2 article of the Spanish Constitution. Currently, there is a wide variety of programs to intervene the deficiencies and needs of the inmates, for example, drug addiction, gender violence offenders, sexual offenders, violent behavior, addiction to gambling, among others. This article aims to bring the treatment work of the prison closer to the reader. The focus of this paper is on briefly explaining three of them, Sexual Assault Control Program (PCAS), Integral Attention to Mentally-ill Persons Program (PAIEM) and Therapeutic and Educational Units (UTEs). Finally, we can add some conclusions about the penitentiary treatment programs, some limitations and possible solutions.
\end{abstract}

Keywords: treatment, prison, programs, sexual offenders, mental disorder, drugs.

\title{
Introducción
}

Si comparamos las políticas y los sistemas penitenciarios a nivel europeo, encontraríamos grandes diferencias en función de cada país. El sistema penal español es relativamente duro, se llevan a cabo muchas medidas de encarcelamiento y, a menudo, éstas son de larga duración, aunque en los últimos años se están aplicando un mayor número de medidas alternativas como trabajos en beneficio de la comunidad, suspensiones y sustituciones de penas y medidas de seguridad (Redondo y Frerich, 2014). Sin embargo, si nos centramos en el tratamiento penitenciario, dichas diferencias casi desaparecen. Lo mismo ocurre con otros países como Estados Unidos y Canadá, este último reconocido internacionalmente como el país de mayor desarrollo en materia de programas de tratamiento y rehabilitación penitenciaria (Redondo, 2017). En este artículo nos centraremos en la realidad actual de los programas de intervención que se aplican en las prisiones españolas, que como hemos avanzado, no son muy diferentes de los que se aplican en las prisiones de los países que nos rodean.

Para contextualizar a nivel legal el tratamiento penitenciario, debemos empezar por citar el artículo 25.2 de la Constitución Española, "las penas privativas de libertad y las medidas de seguridad estarán orientadas hacia la reeducación y reinserción social”. No cabe duda de que el tratamiento es uno de los pilares en los que se sustenta dicha reeducación y reinserción de los/las internos/as dentro de los centros penitenciarios.

El tratamiento penitenciario viene regulado en el Título III de la LOGP de 1979, artículos 59 a 72 y en el título V del Reglamento del 96, artículos 110 a 153. Según el artículo 59 de la LOGP, el tratamiento consiste en el conjunto de actividades directamente dirigidas a la consecución de la reeducación y la reinserción social de los penados y pretende hacer del interno una persona con la intención y la capacidad de vivir respetando la ley penal así como de subvenir a sus necesidades. A tal fin, se procurará desarrollar una actitud de respeto a sí 
mismos y de responsabilidad individual y social con respecto a su familia, al prójimo y a la sociedad en general.

Dicho tratamiento sigue unos principios informadores como, estar basado en el estudio científico del sujeto a tratar, guardará relación directa con un diagnóstico de personalidad criminal y con un juicio pronóstico inicial, será individualizado, complejo, programado, continuo y dinámico (art. 62 de la LOGP).

Si revisamos la literatura, son muchas las teorías que han intentado dar respuesta a la conducta delictiva. En la actualidad podemos destacar dos especialmente importantes, el modelo de RxNxR (riesgo-necesidad-responsividad) de Andrews y Bonta (2010) y el modelo de GLM (Good Lives Model) de Tony Ward (2002) En primer lugar el modelo de Andrews y Bonta fue desarrollado en la década de 1980 y formalizado por primera vez en 1990. Brevemente este modelo intenta explicar las diferencias individuales en el comportamiento delictivo atendiendo a las influencias o refuerzos del más amplio contexto cultural y social, del comunitario y familiar más próximo y de las variables personales del sujeto (biológicas, cognitivas, conductuales, educativas, etc.). Debido a las aportaciones realizadas a la hora de explicar la conducta delictiva, el modelo de Andrews y Bonta (2010) se ha orientado y empleado en las aplicaciones psicológicas para la prevención y tratamiento de la delincuencia, estableciendo tres grandes principios de intervención: el principio de Riesgo, que plantea que los individuos que presentan un mayor nivel de riesgo en factores estáticos, que tienen un nivel de modificabilidad menor (precocidad delictiva, tendencia antisocial), frente a factores dinámicos que si pueden modificarse con mayor facilidad (sistema de creencias, hábitos antisociales, consumo de drogas, influencia del grupo de iguales), requieren intervenciones más intensivas. El principio de Necesidad, que afirma que los factores de riesgo dinámicos que están directamente conectados con la actividad delictiva (tales como hábitos, cogniciones y actitudes delictivas) deben ser los auténticos objetivos de los programas de intervención, ya que se espera que, al superar el sujeto sus necesidades criminógenas, se producirá también un cambio en el nivel de reincidencia, en el sentido de una disminución. Y finalmente el principio de Responsividad, que advierte sobre la necesidad de ajustar y adaptar adecuadamente las intervenciones a las características personales y situacionales de los sujetos para favorecer el éxito del mismo. En segundo lugar, el modelo de tratamiento de Good Lives de Tony Ward (2002) y de sus colaboradores, propone una teoría reeducativa penitenciaria desde el marco legislativo, ético y antropológico de los derechos humanos. Estos derechos ayudan a percibir las necesidades más humanas de los presos, a buscar estilos de vida adecuados -vinculados al cambio- y a favorecer ambientes penitenciarios más respetuosos y humanos con los presos. El modelo de tratamiento de Good Lives propone, de modo muy acertado, pasar de una visión terapéutica y rehabilitadora de los derechos humanos en general, al derecho específico a la educación, la enseñanza y la cultura. El derecho humano a la educación impulsa acciones y no solo políticas pasivas de protección. Se analizan con detalle algunas de las posibilidades educativas de los derechos humanos y del derecho a la educación en los contextos penitenciarios: la condición vulnerable del preso, las posibilidades del cambio personal, la perspectiva crítica de los derechos humanos frente a los derechos particulares de los presos y, por último, el horizonte reeducativo que la idea de la dignidad humana abre en el contexto del modelo de Ward. Este modelo critica las limitaciones del modelo de Andrews y Bonta que se centran en valorar los riesgos del comportamiento delictivo y ofrecen como alternativa una idea basada no solo en "la 
reducción de los factores de riesgo, sino en equiparar a los delincuentes con los recursos para vivir mejores estilos de vida" (Ward y Maruna, 2007 p.89; Ward y Gannon, 2006 p.83).

Hoy en día, no podemos concebir la prisión sin el tratamiento. Sin embargo, las prisiones siguen siendo unas grandes desconocidas en general. Con este artículo se pretende acercar esta realidad a los lectores, donde comentaremos qué funciona y que aspectos necesitan mejorar.

El sistema penitenciario español es un sistema progresivo, que parte del primer grado, continua en segundo, tercero y, por último, la libertad condicional. El Ministerio del Interior y más concretamente la Secretaría General de Instituciones Penitenciarias, han desarrollado a lo largo de estos años un conjunto de programas de tratamiento, que se pueden ver en su página web (citadas en la bibliografía), desde una política moderna y actual con la realidad de las prisiones, adaptándose a los nuevos delitos emergentes, ... En este sentido, podemos encontrar programas específicos de tratamiento en cada uno de los distintos grados, como el Programa de Régimen Cerrado (primer grado), otros que se suelen llevar a cabo en segundo, por ejemplo, programa de Intervención para Agresores (PRIA), Módulos terapéuticos, Juego patológico, Programa de Control de la Agresión Sexual (PCAS), Módulos de respeto, Programa Ser Mujer.es (para la prevención de la violencia de género), Programa de Prevención de Suicidios, Programa de Preparación de los Primeros Permisos, Programa de Intervención de Conductas Violentas (PICOVI), Terapia Asistida con Animales (TACA), el Programa de Atención a Internos con Salud Mental (PAIEM), Tabaquismo, Fuera de red (dirigido a delitos de pornografía infantil), entre otros. En tercer grado, encontramos también programas dirigidos a trabajar la drogodependencia, dirigidos a agresores de violencia de género,...Finalmente señalar otro abanico de programas desarrollados para aquellas personas a las que se les aplican penas y medidas alternativas a la prisión, como el Programa de Intervención Psicoeducativa en Seguridad Vial (PROSEVAL) dirigido a aquellos penados condenados por delitos contra la seguridad del tráfico, Programa en Beneficio de la Comunidad (PROBECO), cuyos objetivos son la sensibilización y reeducación en habilidades sociales, ...

Uno de los interrogantes comunes sobre la eficacia de los tratamientos en prisión, una constante en todos los países occidentales, se denomina el "“What Works?"” (Gendreau y Andrews, 1990). Con esta pregunta se pretende encontrar una respuesta que se acerque a la clave que haga que los programas de tratamiento sean eficaces y eficientes. En la actualidad y dentro de la gran variedad de enfoques psicológicos, modelos y teorías explicativas, no es tarea fácil conocer dichas claves. Parte de la literatura científica está volcada en este objetivo y, a través de diferentes estudios, disponemos de resultados para poder guiar los programas de tratamiento dentro de un contexto, tan peculiar, como son las prisiones.

La gran mayoría de los programas en prisión se realizan de forma grupal, por varios motivos, en primer lugar es necesario que las intervenciones lleguen a la mayor población posible, dada la alta demanda existente $\mathrm{y}$, en segundo lugar, porque el propio grupo participa activamente a nivel terapéutico, se establece una relación de sinergia entre los diferentes miembros del grupo. 
En general, los programas de tratamiento en prisión tienen un enfoque psicológico de tipo cognitivo-conductual, ya que han mostrado una mayor eficacia en diversas medidas evaluativas, lo que incluye también la reducción de la reincidencia delictiva (Gacono et al, 2001; Redondo y Frerich, 2013, 2014; Ward y Eccleston, 2004; Zara y Farrington, 2016). Este enfoque se basa en el principio psicológico general según el cual los procesos cognitivos influyen en la conducta. Así se considera que si se modifican los pensamientos, las actitudes, los razonamientos y las capacidades cognitivas de resolución de problemas interpersonales (lo que también implica mejorar su control emocional y enseñarles nuevas habilidades y conductas), se hace más probable su comportamiento prosocial y una reducción de la frecuencia y gravedad de sus actividades delictivas (Cooke y Philip, 2001; Cullen y Gendreau, 2006)

La duración y temporalidad de los programas es muy variada, pueden ir desde los seis meses hasta los dos años. La extensión temporal es, en ocasiones necesaria, ya que supone provocar cambios profundos en la forma de pensar, sentir y actuar de los internos/as y es necesario que tales cambios se interioricen.

Todos los internos en prisión tienen derecho a participar en los programas de tratamiento, siendo obligación de la Administración Penitenciaria diseñar en cada caso un programa de tratamiento individualizado y dinámico (revisable cada seis meses), incentivando a cada interno para que colabore activamente en su aplicación (art. 12 del RP). El tratamiento, por lo tanto, no puede imponerse coactivamente, sino que es preciso que el interno acepte libremente someterse a él pues exige la cooperación voluntaria del sujeto tratado. La imposición del tratamiento, aparte de atentar contra el principio de respeto a la personalidad de los internos consagrado en el artículo 3 de la LOGP, supone ya su propio fracaso (Manzanares, 1986). Una de las situaciones que nos podemos encontrar es que los internos/as no tengan una verdadera motivación hacia el tratamiento, sino que ésta sea instrumental y el verdadero interés sea conseguir los beneficios penitenciarios, como por ejemplo permisos de salida, un tercer grado, acceder a una comunidad terapéutica, etc. Durante la selección de los internos/as para los diferentes programas es preciso informarles de que el hecho de participar en ellos no significa que vayan a acceder antes a dichos beneficios, evitando así futuras frustraciones durante el desarrollo de los mismos.

A continuación, haremos una pequeña descripción de los siguientes programas: el Programa de Control de la Agresión Sexual (PCAS), el Programa de Atención Integral de Enfermos Mentales (PAIEM) y las Unidades Terapéuticas y Educativas (UTEs). La selección de estos programas específicos de tratamiento se debe, en los dos primeros casos, por la gran alarma social que provocan, ya que existe una gran estigmatización en ambos colectivos, en cuanto a la reincidencia de los agresores sexuales y por la reincidencia violenta en el caso de la población con trastorno mental. Finalmente, hablaremos de las UTEs porque el consumo de drogas es uno de los problemas más importantes en prisiones, se plantea una metodología diferente de intervención y cómo estos programas logran alcanzar unos resultados muy alentadores. 


\section{Programa de Control de la Agresión Sexual (PCAS)}

La violencia sexual es un importante motivo de preocupación social, ya no solo por su coste económico sino por el elevado nivel de sufrimiento humano que lleva asociado (Herrero, 2013). Algunos de estos delitos surgen de la mano del uso de las nuevas tecnologías, como internet, la pornografía infantil, entre otros, tal y como señalan Negredo y Herrero (2016).

Debido a la alarma social que provocan este tipo de delitos, algunos países como Estado Unidos, llevan a cabo una serie de medidas extremadamente severas, como, por ejemplo, la prohibición de residencia en un determinado lugar, la obligación de registrarse en listados públicos de agresores sexuales o de notificar en el nuevo vecindario la condición de delincuente sexual. A pesar de que la opinión pública relaciona este tipo de delitos con una alta tasa de reincidencia en delitos similares, la realidad es distinta. Los delincuentes sexuales presentan, en comparación con otros tipos de delincuentes y de forma general una baja reincidencia y, si reinciden, lo suelen hacer en otro tipo de delitos, no sexuales (Herrero, 2013). En un estudio retrospectivo con internos de prisión llevado a cabo en España, un 8,5\% de los agresores sexuales $(n=82)$ eran reincidentes en el mismo tipo de delito, mientras que en el caso de los delincuentes contra la propiedad $(n=167)$ este porcentaje subía hasta el 37,4\% (Graña, Andreu y Silva, 2009): Los agresores sexuales reincidentes y especializados son una minoría dentro de esta población. Conocer más sobre ellos y sus características nos permitirá en el futuro un mejor diseño de las políticas penitenciarias y poder mejorar la toma de decisiones en relación a esta población.

El tratamiento en prisión de los agresores sexuales se inició en nuestro país en el año 1998. El programa y el procedimiento de intervención se encuentra descrito en un manual adaptado por profesionales de la institución y publicado en la colección de Documentos Penitenciarios ("El control de la Agresión Sexual: programa de intervención en el medio penitenciario").

Este programa está dirigido a internos con delitos sexuales tanto sobre mujeres adultas como hacia menores. Tiene una duración de unos dos años aproximadamente. Se lleva a cabo con pequeños grupos, entre 10 y 15 personas, aunque se pueden hacer sesiones individuales siempre que se considere necesario. Los internos tienen que reconocer los delitos para poder acceder al programa. En él participan varios miembros del equipo técnico, educadores/as, trabajadores/as sociales, juristas y psicólogos/as. El programa se estructura en doce módulos agrupados en dos grandes bloques: toma de conciencia y toma de control.

En el primero de ellos se trata de tomar conciencia de las emociones y conductas que encaminan hacia un comportamiento violento, de forma que se propicie una disminución del nivel de resistencia hacia la admisión del propio comportamiento criminal. Durante el segundo bloque se analiza la propia conducta delictiva y se enseñan y entrenan habilidades dirigidas a la toma de control y prevención de posibles nuevos comportamientos sexuales inadecuados y violentos.

El programa aborda los siguientes contenidos: análisis de la historia personal, distorsiones cognitivas y mecanismos de defensa, conciencia emocional y empatía, comportamientos violentos, educación sexual, modificación del impulso sexual, prevención de recaídas y estilo de vida positivo. 
Este programa suele comenzar explicando varias técnicas de relajación, debido a que las sesiones posteriores suelen ser especialmente difíciles (por ejemplo, trabajar los hechos probados de las sentencias delante del grupo) y los internos tienen que ser capaces de aprovechar al máximo las sesiones y después regresar a los respectivos módulos con un nivel de ansiedad adecuado. Durante el programa se hace una revisión de las historias de vida de los participantes para poder detectar que ha fallado en el aprendizaje, los modelos a imitar, si ha sufrido abusos sexuales o no en la infancia, la educación sexual recibida, el consumo de sustancias tóxicas, etc., para intervenir posteriormente. Los delincuentes sexuales que participan en estos programas suelen ser personas que presentan características psicológicas como una baja empatía y una escasa conciencia emocional. Tienen una gran dificultad a la hora de expresar y reconocer emociones, tanto en sí mismos como en otras personas. Es necesario, por medio del tratamiento, eliminar ciertos mecanismos de defensa que los internos desarrollan en torno al delito sexual, como, por ejemplo: "me decía que no, pero en realidad si quería", "yo estaba bajo los efectos de las drogas y no era yo mismo".

Uno de los puntos clave donde debe de intervenir el programa son las distorsiones cognitivas. Estos mecanismos psicológicos, de naturaleza básicamente cognitiva, podemos definirlos como interpretaciones erróneas de la realidad que llevan al individuo a percibir el mundo de manera poco objetiva, además de disfuncional. Se presentan en forma de pensamientos automáticos y desencadenan emociones negativas que dan lugar a conductas no deseadas o desadaptativas. Es preciso detectarlas y poder sustituirlas por pensamientos alternativos y adaptativos ya que están estrechamente relacionados con el delito sexual y su probabilidad de aparición.

En otros módulos del programa y en otras sesiones se tratan aspectos relacionados con un estilo de vida saludable y positiva, la educación sexual, etc. También si se detectan factores de riesgo, como alguna parafilia, sería necesario atenderla, muchas veces de forma individual con el interno.

Finalmente, el programa de tratamiento se centra en la prevención de las recaídas. A pesar de la alarma social que provoca este tipo de delitos, el nivel de reincidencia en el mismo delito suele ser bajo y más aún cuando se recibe tratamiento (Redondo y Martínez, 2012).

Una vez que se termina el programa PCAS es muy aconsejable administrar algún protocolo o guía de valoración del riesgo de violencia sexual, como por ejemplo el Sexual Violence Risk Scale (SVR-20) desarrollado por Boer, Hart, Kropp y Webster en 1997 o el Risk of Sexual Violence Protocol (RSVP) desarrollado por Hart, Kropp y Laws en 2003, para estimar el riesgo de reincidencia y diseñar un plan de gestión de ese riesgo, especialmente cuando el interno está a punto de volver a la comunidad por algún permiso, libertad condicional o licenciamiento del centro penitenciario por finalización de la condena. El SVR-20 está compuesto por 20 ítems relativos a factores de riesgo, tanto estáticos como dinámicos que se agrupan en tres escalas: funcionamiento psicosocial, delitos sexuales y planes de futuro y, el RSVP, consta de 22 ítems de riesgo estáticos y dinámicos, basados en la revisión de literatura y en la consulta de expertos, referidos a distintas áreas como por ejemplo la historia de delitos sexuales, el ajuste psicosocial o los planes futuros. Estas guías de valoración del riesgo de violencia sexual nos dan información sobre si el riesgo de un determinado interno es bajo, medio o alto y son de especial interés a la hora de valorar los permisos de salida de los 
internos, las posibles progresiones de grado, etc. Finalmente, la toma de decisiones por parte de los diferentes miembros de la Junta de Tratamiento será más adecuada, si además de basarnos en la experiencia o el juicio clínico del profesional, también tomamos en cuenta la información procedente del programa de tratamiento y de las guías de valoración del riesgo.

\section{Programa de Atención Integral de Enfermos Mentales (PAIEM)}

La realidad de las personas con un trastorno mental ha cambiado mucho a lo largo de estos años, según indica Leganés Gómez, S. (2010). El cierre de los centros psiquiátricos supuso un paso adelante para la rehabilitación y mejora de esta población. No obstante, las familias y la propia comunidad no siempre han podido absorber la demanda o la atención que estos sujetos necesitan. Una consecuencia indeseada de esta realidad, de la presencia de personas con trastorno mental en la comunidad, a veces pacientes con trastornos mentales graves y otras dificultades sociales, es que tienen mayores probabilidades de cometer delitos o faltas y ser condenados a una pena privativa de libertad o a una medida de seguridad (Ruiz y Giráldez, 2017).

Las medidas de seguridad deben cumplirse en los hospitales psiquiátricos. En España, actualmente y como tales, solo existen dos centros, uno en Alicante (de hombres y mujeres) y otro en Sevilla (solo de hombres), para absorber la demanda actual de las medidas de seguridad.

Se estima que la prevalencia de trastorno mental en población reclusa es cinco veces más alta que en la población general, según se indica en el estudio PRECA (2011). El origen de los mismos puede ser de lo más variado, podemos clasificarlos en primarios, cuando el origen no está relacionado aparentemente con ninguna otra circunstancia, por ejemplo, una familia estructurada donde aparece una esquizofrenia y secundarios, cuando el trastorno mental emerge como consecuencia de, por ejemplo, una historia más o menos larga de consumo. Además, un elevado número de internos/as suelen padecer de forma simultánea a lo largo del ciclo vital de una adicción y de otro trastorno mental, lo que denominamos patología dual, complicando más su tratamiento y sobre todo su reinserción en la sociedad. Una de las realidades que nos encontramos a la hora de trabajar la reinserción de estos internos es que ellos podrían acceder a recursos, por ejemplo, de salud mental, pero no llegan a ser aceptados debido a su historia de consumo de tóxicos y viceversa. La patología dual todavía no tiene los recursos en la comunidad necesarios para dar respuesta a su demanda.

Para poder mejorar el intercambio de información entre los diferentes profesionales de Instituciones Penitenciarias, como médicos, psicólogos, trabajadores sociales, educadores sociales, etc., y atender las necesidades de esta importante población de internos penitenciarios se creó el Programa de Atención Integral de Enfermos Mentales (en adelante PAIEM). Esta base de datos facilita la consulta, el seguimiento y la atención a todos los internos con enfermedad mental que se encuentren en prisión. Los criterios de inclusión en el PAIEM hacen referencia a la presencia de sintomatología conductual que interfiere en la vida penitenciaria de un interno con trastorno mental grave estabilizado (los enfermos agudos deben en primer lugar ser estabilizados). Por lo tanto, según estos criterios estarían incluidos los siguientes trastornos: trastornos de personalidad, trastornos del estado del ánimo, esquizofrenia y otros trastornos psicóticos, patología dual, etc. Y quedarían excluidos, según 
esta clasificación, los siguientes: deficiencia mental y los trastornos inducidos por el uso de sustancias psicoactivas en exclusiva.

Desde hace pocos años se están creando en las prisiones españolas módulos específicos de Salud Mental, donde se encuentran aquellos internos ya diagnosticados que no logran adaptarse a los módulos ordinarios, por diferentes motivos, como, por ejemplo, necesitar ayuda para la higiene personal, el control de la medicación, etc. Cuando dichos internos están muy deteriorados o se encuentran descompensados, suelen estar en el módulo de Enfermería, donde los cuidados y la supervisión médica son continuos. El resto de los internos con trastornos mentales se encuentran en los módulos restantes, ya sean ordinarios, de respeto o de destinos productivos y con un especial seguimiento por parte de los equipos de tratamiento y los Servicios Médicos.

En los módulos específicos de salud mental, descritos anteriormente, destaca la función de los internos de apoyo que suelen ser formados a través del Programa de Internos de Apoyo, que se realiza en los diferentes centros, no solo para esta función, también para encargarse de la supervisión de aquellos internos con intentos autolíticos, etc. Estos internos se encargan de todas aquellas tareas que el interno/a que tiene el trastorno mental no puede, temas de higiene, la medicación, tarjeta de peculio,..., pero siempre potenciando su independencia. Por ejemplo, si un interno no sabe administrarse el dinero porque nadie le ha enseñado, el objetivo no es que el interno de apoyo se lo administre, sino enseñarle para que lo haga él mismo y prepararles para la vida en libertad.

El funcionamiento de estos módulos puede ser, en ocasiones más laxo, por ejemplo, a la hora de imponer una sanción disciplinaria. Es necesario que los funcionarios, tanto de vigilancia como del equipo técnico, que trabajen en ellos tengan una especial sensibilidad para tratar adecuadamente a los internos con problemas de Salud Mental. Brevemente, en estos módulos sin dejar de lado la seguridad, prima el tratamiento.

Este tipo de población penitenciaria, al igual que sucede con los agresores sexuales, provoca una gran alarma social ya que se suele asociar la salud mental a delitos de especial gravedad, como asesinatos, homicidios, etc. pero los estudios actuales muestran una relación un tanto confusa (Loinaz, Echeburúa e Irureta, 2011). No podemos obviar que determinados trastornos, como por ejemplo la esquizofrenia, cuando no están tratados, se encuentran en una fase aguda, presentan un consumo de sustancias tóxicas, pueden generar dificultades al interno de adaptación y conducta al perder el contacto con la realidad y esto puede aumentar la probabilidad de cometer un delito violento (Esbec y Echeburúa, 2016). Se hace imprescindible una adecuada detección de las diversas patologías o trastornos mentales y realizar una gran labor de prevención. De esta manera se lograría reducir la vulnerabilidad de este colectivo, así como su estigmatización (Echeburúa y Loinaz 2011).

Debido a los problemas específicos de este grupo de internos, en los centros penitenciarios y a la hora de estudiar la separación interior, los permisos de salida, su reinserción social, entre otros, es interesante emplear, para prevenir los problemas de comportamiento violento y antinormativo, los protocolos o guías de valoración del riesgo existentes a tal efecto. Para esta población podríamos aplicar el Historical Clinical Risk (HCR-20 V3) desarrollado por Douglas, Hart, Webster y Belfrage, ya que está diseñado para la gestión de estos problemas 
en poblaciones penitenciaria y psiquiátrica. Esta guía consta de 20 ítems, tanto estáticos como dinámicos, y que están clasificados en factores históricos, clínicos y de gestión futura del riesgo. Después de aplicar dicha guía de valoración del riesgo de violencia obtendremos una valoración estimada del riesgo en tres niveles: bajo, medio o alto que permitirán diseñar medidas de gestión adecuadas a esos riesgos. El HCR-20 V3, es un instrumento del juicio profesional estructurado, gracias al cual el profesional tomará sus decisiones utilizando su experiencia y formación profesional ayudado por la guía de valoración del riesgo. Actualmente existen estudios donde queda patente el mayor grado de eficacia del juicio profesional estructurado frente al juicio clínico no-estructurado (Andrés-Pueyo y Redondo 2007).

Por último, señalar, en relación con los internos que tienen problemas relevantes de salud mental, que la transición de la prisión a la vida en la comunidad al finalizar la condena, su reinserción, al fin y al cabo, suele ser más complicada que en la población general (Ruiz y Giráldez, 2017). Con esta población es necesario un buen trabajo en equipo entre los diferentes servicios, no solo sanitarios, para que continúen con las citas y su tratamiento por otros servicios de Salud Mental, las familias o los centros de acogida para que mantengan los hábitos, la adherencia al tratamiento, la gestión del tiempo en libertad a través de talleres, búsqueda activa de empleo y, en el caso de que haya un consumo de tóxicos, continuar el contacto con los servicios de atención de las drogodependencias de su zona de residencia, etc. De esta manera podremos reducir la probabilidad de reincidencia delictiva de esta población.

\section{Unidades Terapéuticas y Educativas (UTEs)}

El consumo de drogas es uno de los principales problemas en la sociedad y también representa un grave problema en las prisiones. Por esta razón, son muchos los recursos que se invierten para intentar erradicarlo desde la propia Secretaría General de Instituciones Penitenciarias, así como las diversas ONGs, asociaciones, etc. que trabajan de manera simultánea dentro de las prisiones, como por ejemplo Proyecto Hombre, Centros Provinciales de Drogodependencias (CPDs en la Comunidad de Andalucía), entre otros.

La denominada "metodología de la UTE", iniciada en el Centro Penitenciario de Villabona (Asturias), identifica a un programa que se desarrolla desde 1992 para el tratamiento de los problemas de drogodependencias de los internos penitenciarios. Se ha desarrollado y generalizado a otros centros debido a su eficacia y eficiencia en la recuperación personal de las personas ingresadas en centros penitenciarios y su preparación para la reincorporación a la sociedad. En la actualidad hay muchas UTEs funcionando en los diferentes centros penitenciarios en toda España.

Podemos decir que las UTEs son módulos independientes, dentro de un centro penitenciario, que albergan a internos en un programa integral para el tratamiento de las drogodependencias. Se constituye un espacio socioeducativo y terapéutico, libre de las interferencias que genera la droga, fomentando cambios de los hábitos, actitudes y valores de los internos/as residentes y creando un ambiente dinámico y personalizador, en el que se adquiere mayor eficacia la intervención multidisciplinar dirigida a la normalización y reincorporación social de los internos/as. 
Las UTEs están basadas en los grupos terapéuticos y en un Equipo Multidisciplinar. El grupo terapéutico o grupo de autoayuda es el eje central de la UTE. Cada grupo está compuesto por 12-15 internos. El grupo terapéutico es el espacio de comunicación donde el interno va a mostrar todas sus carencias y déficits de socialización, a través de la revisión, reflexión y confrontación con el resto de los miembros del grupo. Se establecen imposiciones y tareas para evitar o reforzar determinadas actitudes y conductas. Se celebra una sesión semanal como mínimo dirigida por un profesional.

El Equipo Multidisciplinar que actúa en el programa UTEs lo forman profesionales de Instituciones Penitenciarias de todas las áreas de intervención: psicólogos, educadores, vigilancia, trabajadores sociales, monitores, maestros, sanitarios, etc. Todos estos profesionales desarrollan actividades terapéuticas y educativas a nivel grupal e individual. Adquiere especial relevancia el área de funcionarios de vigilancia, que, al representar la primera línea de la intervención dentro de la institución penitenciaria, permite el seguimiento del proceso terapéutico de los internos/as durante las veinticuatro horas del día.

Los internos que están en este programa se vinculan a él por medio de un contrato terapéutico, que es un documento escrito que recoge los compromisos, derechos y deberes de ambos. La escuela, de asistencia preferente, es uno de los pilares de la UTE, al constituirse como medio estimulante, atractivo y dinámico que ayuda de forma fundamental a conseguir el nivel de concienciación y normalización social. Otras actuaciones propias de este programa buscan provocar cambios importantes en los hábitos, actitudes y valores y entre ellas se incluyen los talleres de salud, los formativos-ocupacionales (cristales, cerámica, debate, informática, dibujo, etc.) los formativos para el empleo, las actividades deportivas, culturales, de ocio y tiempo libre; los cursos formativos en el exterior, las salidas terapéuticas, los campos de trabajo y los permisos de salida.

Al igual que ocurre con el resto de los programas, el interno no siempre está en el momento idóneo para iniciar el tratamiento, de ahí la importancia de la motivación por parte de los diferentes profesionales. Con la ayuda del llamado Modelo Transteórico del cambio de Prochaska y DiClemente (1982), aplicado a la situación del interno, de sus emociones, actitudes, etc. se puede atender a los estadios psicológicos que nos indican cómo, cuándo y por qué cambian las personas. Según este modelo estos estadios son: precontemplación, contemplación, acción y mantenimiento. Según este modelo en la primera fase, el sujeto aún no es consciente de que tenga un problema, en este caso con el consumo de sustancias. En la segunda fase, empieza a darse cuenta de que tiene un problema, pero todavía no ha tomado la decisión de hacer algo al respecto. Es en la tercera fase de acción donde ya existe una verdadera implicación en el tratamiento de la drogodependencia. Y finalmente, la fase de mantenimiento, consistiría en que aquellos logros conseguidos, la abstinencia de las diferentes sustancias tóxicas, se mantuviesen a lo largo del tiempo. Según lo anterior, es necesario evaluar el momento puntual, el estadio en que se encuentra el interno/a para saber si intervenir mediante entrevistas individuales para que sea consciente del problema que tiene, posteriormente para motivarle al tratamiento y cuando se encuentre en una fase de acción incluirle en el programa de drogodependencias y la prevención de recaídas.

Además, no debemos olvidar que cuando se ha tenido un problema de drogodependencia es vital continuar con los seguimientos por los diferentes recursos que existen en la comunidad, 
ya sean los Centros Provinciales de Drogodependencias (CPD), Proyecto Hombre, Cruz Roja, entre otros. Es importante la colaboración de los centros penitenciarios con los diferentes recursos antes mencionados, para potenciar el mantenimiento de la abstinencia, continuar con el Programa de Mantenimiento de Metadona, en aquellos casos que lo requieran.

Otro de los recursos que dispone instituciones penitenciarias para el tratamiento de la drogodependencia es la derivación a una Comunidad Terapéutica, a través de un tercer grado, artículo 182 del Reglamento Penitenciario, aprobado por Real Decreto 190/1996 de 9 de Febrero, en el cual se indica "El Centro Directivo podrá autorizar la asistencia en instituciones extra-penitenciarias adecuadas, públicas o privadas, de penados clasificados en tercer grado que necesiten un tratamiento específico de deshabituación de drogodependencias y otras adicciones, dando cuenta al Juez de Vigilancia". De esta manera, aquellos internos/as que lleven una evolución en la UTE pueden ser candidatos a continuar su tratamiento en alguna de estas comunidades.

\section{Conclusiones}

La literatura sobre el tratamiento en prisión es cada vez mayor, tanto a nivel europeo como en otros países como Canadá, pionero por su gran desarrollo en programas de tratamiento y rehabilitación con delincuentes.

El tratamiento penitenciario es fundamental para conseguir los objetivos de reeducación y de reinserción social que emanan de la Constitución Española, así como su reflejo en la LOGP de 1979 y en el RP. de 1996. Dicho tratamiento debe ser individualizado, dinámico, continuo y entre otros aspectos, voluntario. En ningún momento podemos coaccionar a los internos/as para que participen en ellos. Los internos/as tienen que darse cuenta de sus carencias y necesidades, las cuales les han llevado a cometer uno o varios delitos y que su tratamiento sería un factor de protección de cara a una posible reincidencia. En estos casos, se hace imprescindible la labor de motivación al tratamiento por parte de los diferentes profesionales de instituciones penitenciarias. Dicha labor consiste en la concienciación por parte del interno/a de su problemática y que posteriormente pueda tomar la decisión libremente de participar en los programas específicos de tratamiento que se ajusten a su situación.

Los estudios cuyos objetivos son conocer cuáles son las claves de los tratamientos más eficaces en prisión, lo que denominamos "What Works", nos muestran que son aquellos con un enfoque cognitivo-conductual, centrados en variables dinámicas y, por lo tanto, modificables, preferentemente en formato grupal, con una duración variada, desde los seis meses hasta los dos años, etc. Sin embargo, además de que los internos/as realicen los programas con un buen desempeño, debemos ir más allá y preparar su puesta en práctica en libertad, conociendo cual es el medio al que regresa, si es o no prosocial, si existe o no apoyo social, etc. Este último aspecto va a condicionar la probabilidad de reincidencia en el futuro.

Brevemente se han comentado tres de los programas específicos de tratamiento que se aplican en la actualidad en muchos de los centros penitenciarios españoles. El programa PCAS, dirigido a agresores sexuales, tanto de mujeres adultas como menores, es un programa intenso, con una duración de dos años, donde se trabajan doce módulos, agrupados en dos 
bloques: toma de conciencia y toma de control. Para poder participar en el programa es necesario que los internos reconozcan el delito. Este programa muestra buenos resultados en cuanto a reincidencia. En diferentes estudios encontramos que el nivel de reincidencia de estos internos es bajo y, si reinciden, suele ser en otro tipo de delitos, como por ejemplo delitos contra la propiedad.

El programa del PAIEM, permite una mayor adecuación de los internos/as diagnosticados de trastorno mental en las prisiones. Encontramos tres niveles, aquellos sujetos que no se adaptan a la vida en prisión, para los cuales se crean módulos específicos de Salud Mental, aquellos internos/as agudos o descompensados por su trastorno mental que residen en el módulo de enfermería, donde la atención médica es constante y, por último, aquellos que se adaptan a los módulos ordinarios sin problemas. Este programa ha mejorado notablemente la calidad de vida de estos internos/as en las prisiones, donde el contacto con los profesionales adquiere otra perspectiva, igual que la relación con los familiares. Por ello, se considera muy necesario que quienes trabajen directamente en estos módulos tengan una especial sensibilidad para tratar con este tipo de población.

La metodología propia de la UTE tiene su origen en Villabona (Asturias) en el año 1992. Este nuevo método de trabajo abarca cuatro campos de intervención: individual, grupal, familiar y comunitaria. La actuación terapéutica y educativa contempla las particularidades de la persona interna y de su ambiente en prisión y fuera de la misma. Los instrumentos que conforman el modelo de Educación Integral que representa la UTE son el Grupo Terapéutico, la Escuela, los talleres ocupacionales y formativos, la asistencia sanitaria, los permisos y las salidas terapéuticas y las actividades cotidianas, culturales, de ocio, etc.

Señalar la importancia de la aplicación de las diferentes guías y protocolos de valoración del riesgo para que los profesionales tengan una mayor información a la hora de tomar decisiones relevantes como permisos de salida, terceros grados, etc. Hemos señalado las guías en torno a delitos sexuales como son el Manual para la valoración del riesgo de violencia sexual (SVR20) o el Protocolo para la valoración del riesgo de violencia sexual (RSVP), en relación a internos/as con trastorno mental aplicaríamos el Historical Clinical Risk (HCR-20 V3). Otras escalas de gran utilidad en contextos forenses y penitenciarios son el Manual para la valoración del riesgo de violencia contra la pareja (SARA), Manual para la valoración estructurada de riesgo de violencia en jóvenes (SAVRY), etc. dependiendo de cada caso en particular. Destacar que en las prisiones catalanas han incorporado el protocolo y las escalas de Valoración del Riesgo de Violencia para Internos (RISCANVI). Este instrumento fue creado por el Dr. D. Antonio Andrés Pueyo, la Dra. Dña. Karin Arbach Lucioni y el Dr. D. Santiago Redondo Illescas, del Grupo de Estudios Avanzados de Violencia de la Universidad de Barcelona. Les permite de una manera sencilla obtener datos sobre la valoración del riesgo de cada interno. Sería de gran ayuda que esta iniciativa catalana se extrapolara al resto de España, para facilitar la labor de los diferentes profesionales.

Podemos llegar, a partir de lo expuesto, a las siguientes reflexiones. En primer lugar, no cabe duda de que poco a poco se ha realizado un importante avance en el tratamiento penitenciario y que debe de continuar en el futuro. Para ello es necesario un mayor número de investigaciones que revisen dichos tratamientos, sus contenidos, su eficacia y eficiencia, etc. para que puedan seguir mejorando. En segundo lugar, decir que, gracias a la implantación de 
los Módulos de Respeto, los/as internos/as mantienen o establecen un mayor número de actitudes y conductas prosociales que les ayudará de cara a su reinserción en la sociedad. Estos módulos llegan a la mayoría de los internos/as y además les permiten una participación más activa en el día a día, colaborando en las actividades, en determinadas tomas de decisiones, ....En tercer lugar, la temporalidad de algunos programas puede considerarse como una limitación, ya que provoca que accedan una proporción menor de internos/as, como ocurre en el Programa de Control de la Agresión Sexual (PCAS), aunque hay que recordar que no todos los internos con este delito lo solicitan, entre otras razones porque no reconocen el delito. Algunas de las posibles soluciones para este hándicap es el traslado del interno por motivos tratamentales a otro centro donde el programa estuviera a punto de comenzar.

No podemos negar que, como opinan Redondo (2017) y otros muchos autores, la prisión no es el lugar más adecuado para recibir tratamiento. Sin embargo, a día de hoy, la prisión sigue siendo necesaria hasta que se apueste firmemente por otras medidas alternativas. Los principales objetivos a seguir deben ser los siguientes, una buena separación interior y una adecuada clasificación, para evitar la contaminación entre los propios internos/as, por ejemplo entre primarios y reincidentes; enseñar y mantener actitudes y hábitos de competencia social, a través de los módulos de respeto; emplear el tiempo en prisión de manera productiva a través de la escuela, el trabajo, el deporte, los diferentes cursos de formación laboral y ocupacional; la implicación en los programas de tratamiento específico $\mathrm{y}$, finalmente, conseguir a través de los distintos beneficios penitenciarios (permisos, salidas programadas, etc.) una adecuada reincorporación a la sociedad. Para alcanzar esta última fase de reinserción es necesario establecer una mayor colaboración entre los centros penitenciarios y los recursos existentes en la comunidad (Salud Mental, drogodependencias, centros de acogida, etc.), De esta manera, aumentaremos aquellos factores de protección de los internos/as e intentaremos reducir aquellos factores de riesgo de cara a su reinserción social, potenciando el desistimiento del delito.

\section{Referencias}

Andrés Pueyo, A. y Redondo Illescas, S. (2007). Predicción de la violencia: entre la peligrosidad y la valoración del riesgo de violencia. Papeles del Psicólogo. Vol. (28)3, 157-173.

Andrews, D.A. y Bonta, J. (2010). The psychology of criminal conduct (5rd ed.) New Providence, NJ: LexisNexis Matthew Bender.

Cervelló, V. (1996) "Restricción de beneficios penitenciarios en el código penal de 1995”. Cuadernos jurídicos $\mathrm{n}^{\circ}$ 42, p. 40.

Cervelló, V (2006). Derecho Penitenciario. 1era ed. Valencia. Editorial Tirant lo Blanch.

Choe, J. Y., Teplin, L.A. y Abram, K.M. (2008). Perpetration of violence, violent victimization, and severe mental illness: balancing public health concerns. Psychiatric Services, 59, 153-164. 
Cooke, D.J. y Philip, L. (2001). To treat or not to treat? An empirical perspective. En C. R. Hollin (ed.), Offender Assessment and Treatment (pp. 17-34), Chichester (Reino Unido): Wiley.

Cullen, F. T, Gendreau, P. (2006) Evaluación de la Rehabilitación Correccional: política, práctica y perspectivas. En R. Barberet y J. Barquín, Justicia penal siglo XXI: Una selección de Criminal Justice 2000, (pp. 275-348). Granada. Editorial Comares.

DiClemente, C.C y Prochaska, J. O. (1982). Self-change and therapy change of smoking behavior: A comparison of processes of change in cessation and maintenance. Adicctive Behavior 7(2), 133-142.

DiClemente, C.C, Garay M. \& Gemmell, L. (2008). Motivational enhancement. En M. Galanter, \& H. D. Kleber (Eds.). The American Psychiatric Publishing Textbook of Substance Abuse Treatment (4th ed). (pp. 361-371). Arlington (US): American Psychiatric Publishing, Inc.

DiClemente, C.C, Schulundt, D. \& Gemmell, L (2004). Readiness and Stages of Change in Addiction Treatment. The American Journal On Addictions, 13(2), 103-109.

Echeburúa, E. y Fernández-Montalvo, J. (2006) Evaluación del programa de tratamiento en prisión para agresores en el ámbito familiar. Documento no publicado, Dirección General de Instituciones Penitenciarias.

Echeburúa, E. y Loinaz, I. (2011). Violencia y trastornos mentales. Violencia y psicología comunitaria. Aspectos psicosociales, clínicos y legales (pp. 1-22).

Esbec, E. y Echeburúa, E. (2016). Violencia y esquizofrenia: un análisis clínico-forense. Anuario de Psicología Jurídica. Volumen 26, Issue 1, 70-79.

Gacono, C Niebering, R. Owen, A., Rubel, J. y Bodholdt, R. (2001) Treating conduct disorder, antisocial, and psychopatic personalities. En J.B. Ashford, B, Sales y W. Reid (ed), Treating adult and juvenile offenders with special needs (pp. 99-130). Washington, DC: American Psychological Association.

Gallego, M., Cabrera P. J., Ríos J. C., Segovia, J. L. (2010). Andar 1 Km en línea recta: la cárcel del siglo XXI que vive el preso. Madrid. Universidad Pontificia de Comillas.

Gendreau, P y Andrews, D.A. (1990). Tertiary Prevention: What the Meta-analyses of the Offender Treatment Literature Tell Us About "What Works". Canadian Journal of Criminology. Vol: 32 Issue: 1. 173-184.

Graña, J.L., Andreu, J.M. y Silva, T. (2009). Evaluación del riesgo de reincidencia en España. Madrid: Ministerio del Interior. 
Grupo PRECA (2011). Informe Prevalencia de Trastornos Mentales en Centros Penitenciarios Españoles. (Estudio PRECA). Barcelona. Ministerio del Interior.

Herrero, O. (2013). ¿Por qué no reincide la mayoría de los agresores sexuales? Anuario de Psicología Jurídica, 23, 71-77.

Herrero, O., Negredo, L. (2015). Fuera de la Red: Programa de Intervención frente a la delincuencia sexual con menores en la red. Madrid. Ministerio del Interior. Secretaría General Técnica.

Leganés Gómez, S. (2010). Enfermedad mental y delito (Perspectiva jurídica y criminológica). La Ley Penal, 76, 1-40.

Loinaz, I., Echeburúa, E. e Irureta M. (20011) Trastornos mentales como factor de riesgo de victimización violenta. Behavioral Psychology/psicología conductual. Vol. 19, № 2, 421-438.

Manzanares Samaniego, J.L. (1986). Comentarios a la legislación penal, tomo VI, Vol. II, Madrid. Editorial Revista de Derecho Privado.

Martínez Catena, A., y Redondo, S. (2016). Etiología, prevención y tratamiento de la delincuencia sexual. Anuario de Psicología Jurídica, 26, 19-29.

Negredo, L. y Herrero, O. (2016) Pornografía infantil en Internet. Papeles del Psicólogo. Vol. 37(3), 217-223.

Nistal Burón, J. y Fernández Arévalo, L. (2015). Derecho penitenciario. Pamplona. Thomson Reuters. Aranzadi.

Pérez-Ramírez, M., Redondo-Illescas, S., Martínez-García, M., García-Forero, C., y AndrésPueyo, A. (2008). Predicción de riesgo de reincidencia en agresores sexuales. Psicothema, 20(2), 205-210.

Redondo, S. (2017). Evaluación y tratamiento de delincuentes Jóvenes y adultos. Madrid. Ed. Pirámide.

Redondo, S y Frerich, N. (2013). Offender Rehabilitation in Europe. Applications, achievements and challenges. En A. Khun, P Margot, M. F. Aebi, C Schwarzenegger, A. Donatsch y D. Jositsch (eds). Kriminologie, Kriminalpolitik und strafrechtausinternationaler perspective (pp. 879-894). Berne: StämfliVerlag AG Bern.

Redondo, S y Frerich, N. (2014) Crimen and Justice reinvestement in Europe: Posibilities and Challenges. Victims and Offenders: An International Journal of Evidence-based Research, Policy and Practice, 9(1), 13-49. 
Redondo-Illescas, S. y Martínez Catena, A. (2012). Tratamiento y cambio terapéutico en agresores sexuales. Revista Española de Investigación Criminológica. Artículo 8, Número 9, 1-25.

Ruiz, S. y Giráldez, P. I. (2017) Reincidencia delictiva tras cumplimiento de medida de seguridad en Hospital Psiquiátrico Penitenciario. X Congreso (Inter)Nacional de Psicología Jurídica y Forense. Sevilla.

Vicens, E. (2006) Violencia y enfermedad mental. Revista Española de Sanidad Penitenciaria. Vol 8, N³, 95-99.

Ward, T. (2002) Good lives and the rehabilitation of offenders: promises and problems. Agression and Violent Behavior, 7, 513-528.

Ward, T. y Brown, M. (2004) The Good Lives Model and conceptual issues in offender rehabilitation. Psychology, Crime \& Law. 10(3), 243-257.

Ward, T y Eccleston, L. (2004), Risk, responsivity, and the treatment of offenders: introduction of the special issue. Psychology, Crime \& Law, 10(3), 223-227.

Ward, T. and Gannon, T (2006). Rehabilitation, etiology and self-regulation: The Comprehensive good lives model of treatment for sexual offenders. Agression and Violent Behavior 11(1), 77-94.

Ward, T and Maruna, S. (2007). Rehabilitation. London. Taylor \& Francis.

Ward, T. y Siegert, R. J. (2002). Toward a comprehensive theory of child sexual abuse: a theory Knitting perspective. Psychology, Crime and Law, 9, 319-351.

Zara, G. y Farrington, D. P. (2016). Criminal Recidivism: Explanation, prediction and prevention. New York, NY; Routledge, Tylor and Francis Group.

\section{Páginas web consultadas}

Instituciones penitenciarias (2018). Programa Específico en Módulos Terapéuticos. Secretaria General de Instituciones Penitenciarias. Ministerio del Interior. Gobierno de España. Recuperado de http://cort.as/-IKcw

Instituciones penitenciarias (2018). Programa Específico de Agresores Sexuales. Secretaria General de Instituciones Penitenciarias. Ministerio del Interior. Gobierno de España. Recuperado de http://www.institucionespenitenciarias.es/

Instituciones penitenciarias (2018). Programa Específico de Enfermos Mentales. Secretaria General de Instituciones Penitenciarias. Ministerio del Interior. Gobierno de España. Recuperado de http://www.institucionespenitenciarias.es/ 